\title{
Modelo didÁCTICO, CON El USO de las TIC, PARA LA FORMACIÓN MATEMÁTICA DE INGENIEROS
}

\author{
Didactic MOdel, With THE USE OF TIC \\ FOR MATHEMATICS EDUCATION OF ENGINEERS
}

\author{
${ }^{1}$ Roxana Cabrera Puig, ${ }^{2}$ Alicia María Vitale Alfonso \\ 1 "Granma", Departamento de Matemática, Micro X Alamar, La Habana del Este, La Habana, Cuba \\ ${ }^{2}$ Facultad de Economía, Universidad de La Habana, La Habana, Cuba
}

Recibido: 24/0772018 - Aprobado: 28/08/2018

\section{RESUMEN}

El uso acelerado de las Tecnologías de la Información y las Comunicaciones (TIC) dentro del proceso docente educativo, ha transformado el modo de trabajar de los educadores, la forma de aprender de los educandos y la manera de pensar de ambos implicados. La enseñanza de la matemática superior se ha visto revitalizada con estas transformaciones y es por ello que para el apoyo de las clases de esta asignatura se ha analizado, por parte de los educadores que la imparten, cómo integrar un modelo didáctico en el aprendizaje con el uso de las TIC, planteándose como objetivo la elaboración de un modelo didáctico que integre las TIC para perfeccionar la formación matemática del futuro ingeniero. En el desarrollo de la investigación se emplea un conjunto de métodos, entre los que se destacan: el análisis documental, análisis y síntesis, inducción y deducción, históricológico, modelación, experimento pedagógico y métodos estadísticos. El modelo didáctico que se propone tiene una estructura que favorece el trabajo independiente, haciendo uso de variados medios de enseńanza para elevar las estrategias de aprendizaje de los educandos, con el fin de que éstos puedan organizar mejor el tiempo y desarrollar conocimientos, hábitos, habilidades, cualidades y valores. El mismo incluye un sitio web que sirve de soporte material para el estudio del tema de integrales que se imparte en la institución. Este modelo fue sometido a experimentación pedagógica durante dos años de instrucción con grupos de primer ańo de la carrera de ingeniería mecánica.

Palabras clave: formación matemática, modelo didáctico, TIC.

\section{Abstract}

The accelerated use of Information Technology and Communications (ICT) in the educational process, has transformed the way teachers work, how to learn from the students and the way of thinking of both involved. The teaching of higher mathematics has been revitalized with these changes and that is why to support classes of this subject has been

\footnotetext{
${ }^{1}$ alicia@fec.uh.cu, orcid.org/0000-0002-4505-8981

2 alicialfec.uh.cu, orcid.org/0000-0002-4505-8981
} 
discussed by educators that teach how to integrate a didactic model of learning with the use of ICT considering the objective of developing a teaching model that integrates ICT for further training of future mathematical engineer. In developing research uses a set of methods among which are: document analysis, analysis and synthesis, induction and deduction, Historical-Logical, Modeling, and Statistical Methods pedagogical experiment. The proposed educational model has a structure that encourages independent work using varied means of teaching strategies to enhance student learning in order that they can better organize the time and develop knowledge, habits, skills, qualities and values. It includes a website that serves as a support material for the study of the topic of Integrated taught at the institution. This model was subjected to experimental teaching for two years of instruction with groups of first year mechanical engineering career.

Keywords: mathematical formation, model didactic, TIC.

\section{INTRODUCCIÓN}

En el proceso docente educativo se han incorporado diversas alternativas para el aprendizaje. Una de ellas es el empleo de sitios web para lograr que se estimule la capacidad creativa de los educandos. En este caso, la matemática superior es una asignatura que está diseñada para que los educandos modelen el objeto o fenómeno matemático que se estudie. Es decir, proveer a éstos de los contenidos matemáticos indispensables que le permitan hacer análisis geométricos y cuantitativos de los fenómenos relacionados con su especialidad y contribuir de esta manera al desarrollo de su formación matemática.

Es por ello, que, para los educadores que profesan esta asignatura, el desafío consiste en prepararse para diseñar nuevos entornos de interacción virtual en función del aprendizaje de los educandos y estimular el papel protagónico de los mismos, con el propósito de perfeccionar la formación matemática integrando el trabajo con un sitio web para facilitar la búsqueda de información de manera que puedan realizar tareas investigativas y apropiarse por sí solos de nuevos conocimientos.

Un sitio web puede insertarse de diversas maneras en el proceso docente educativo de la matemática superior:

Como apoyo para las tareas que a diario se le pueden presentar a educadores y educandos.
- Como ayuda en la búsqueda de información dispuesta y en existencia a educadores y educandos.

- Como herramienta para procesar información y realizar ejercicios que desarrollen su formación matemática.

En esta propuesta, el sitio web es el soporte de medios de enseńanza a través del cual se propicia la formación matemática esperada. Es decir, el modelo didáctico incluye todos los componentes del proceso docente educativo, pero hace énfasis en la actuación de educadores y educandos mediante un sitio web, como medio integrador y estimulador del aprendizaje de la matemática superior.

\section{Desarrollo}

La matemática superior se concibe y estructura con un enfoque de sistema. Toda la actividad que desarrollan los educandos en esta asignatura va dirigida en primer lugar, a modelar el objeto o fenómeno que se estudie. Es decir, a expresar en el lenguaje de la matemática las principales relaciones que caracterizan el objeto o fenómeno, realizar los cálculos necesarios e interpretar los resultados. Esta asignatura es considerada indispensable en la preparación de profesionales que impulsan, con su gestión, el desarrollo científico técnico del país. 
En un artículo titulado "La enseñanza de la matemática en el umbral del siglo XXI”, (Delgado, 2009), expresa tres ideas fundamentales:

1. Hay que fundamentar desde el punto de vista psicológico y didáctico cómo usar la computadora para que constituya un aprendizaje cualitativamente superior.

2. Hay que hacer una revisión de los contenidos del proceso de enseñanza, de aquellas habilidades que deberán formarse con y sin el uso de estas tecnologías.

3. Hay que combinar los métodos deductivos con los experimentales para que los educandos investiguen, sugieran hipótesis, formulen definiciones y teoremas, vinculando lo inductivo-heurístico con los deductivo-lógicos a partir del uso de la computadora.

Teniendo en cuenta estas argumentaciones, se considera como Modelo didáctico, con el uso de las TIC, para la formación matemática: "La representación simplificada (que propone la combinación de la presencialidad y la no presencialidad) de la relación entre los sujetos del proceso docente educativo utilizando especialmente las tecnologías de la información y redes de comunicación mediante la producción de materiales didácticos para el estudio y una gran variedad de medios de enseñanza poniendo énfasis en el perfeccionamiento del autoaprendizaje para incidir en el desarrollo integral de la personalidad de los educandos".

Para ello no basta con que el modelo exista y esté correctamente definido, es necesario que los educadores y educandos utilicen las TIC que apoyan el proceso docente educativo, de manera que se logre perfeccionar la enseñanza de la matemática superior y la forma de organizar la estructura del conocimiento teniendo en cuenta la sinergia de los componentes del modelo didáctico.

La estructura, puede representarse en el esquema siguiente:

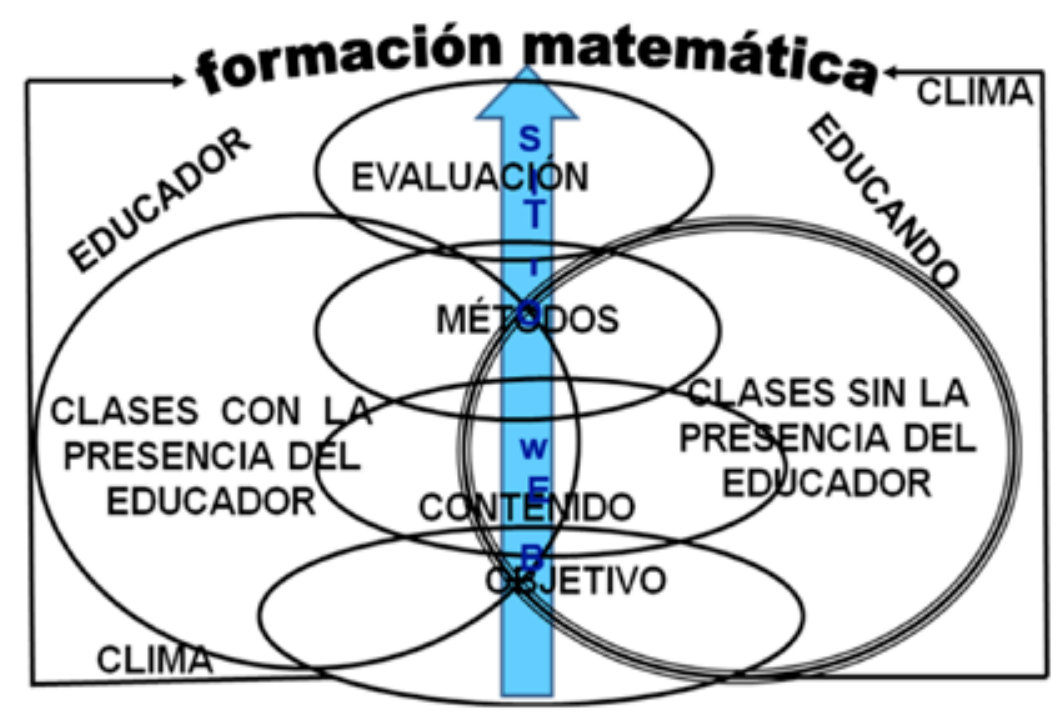

Fig. 1 Estructura del modelo didáctico, con el uso de las TIC, para la formación matemática.

Para facilitar la comprensión de esta estructura se considera que:

Los objetivos: determinan el resto de las componentes de dicho proceso. Es el que responde a la pregunta de ¿para qué enseñar y aprender con un modelo didáctico, con el uso de las TIC, para la formación matemática? La respuesta vendrá dada por los objetivos con que se emplee el sitio web para elevar la formación matemática. 
Los contenidos: constituyen la base para lograr los objetivos, incluye los conceptos, teoremas, algoritmos, las habilidades y cualidades que configuran la formación matemática de los educandos. Es el que responde a la pregunta de ¿qué enseñar y aprender con un modelo didáctico, con el uso de las TIC, para la formación matemática?

Como para este modelo didáctico es vital el contenido que el educando va a adquirir para propiciar la formación matemática el educador se organizará el proceso de asimilación teniendo en cuenta los métodos de enseñanza. En este modelo se emplean de forma combinada en dependencia de los objetivos y el contenido de la enseñanza. Sin embargo, el trabajo independiente es el principal, ya que, mediante el mismo, con una buena orientación del educador, el educando puede vencer los objetivos propuestos y adquirir los conocimientos, habilidades, cualidades y valores que contribuyen a elevar su formación matemática. Es el que responde a la pregunta de ¿cómo enseñar y cómo aprender con un modelo didáctico para la formación matemática? Para el logro de este propósito, en este modelo didáctico para alcanzar el aprendizaje, se precisa la realización de las tareas docentes que van dirigidas a elevar la formación matemática, y que a su vez favorezcan el desarrollo intelectual de los mismos.

Para contribuir a ello se utiliza en unidad dialéctica, como variados medios de enseñanza, "todo objeto real, sustituto material de un objeto real o ideal, capaz de portar el contenido de la enseńanza" (Colectivo de autores, 2009). Es el que responde a la pregunta ¿con qué enseñar y aprender en un modelo didáctico, con el uso de las TIC, para la formación matemática? El sitio web en el modelo didáctico es el soporte de otros medios de enseñanza, puede ser utilizado por educadores y educandos como medio para el aprendizaje y como medio para la investigación y la resolución de problemas ya que brinda la posibilidad de que pueda el educando consultar diferentes bibliografías en formato digital, comprobar diversos resultados haciendo uso de los asistentes matemáticos.

La implementación de un modelo didáctico para la formación matemática que integre las TIC mediante un sitio web implica un cambio ligero en la forma de presentar los ejercicios para la búsqueda de solución por parte de los educandos, es por ello, que se utilizarán como formas de organización de la enseñanza: las clases con la presencia del educador (presenciales) y sin la presencia del educador (no presenciales). En este modelo se hace necesario responder al interrogante ¿cómo organizar el enseñar y aprender con un modelo didáctico, con el uso de las TIC, para la formación matemática?

Las clases con la presencia del educador, son para promover y motivar la búsqueda investigativa de los educandos durante las clases sin la presencia del educador, de manera que cuando utilicen el sitio web, dando solución a las tareas orientadas, puedan así desarrollar su independencia cognoscitiva y sean capaces de apropiarse de nuevos conocimientos y desarrollar hábitos, habilidades, cualidades y valores. Las clases sin la presencia del educador (no presenciales): exigen la preparación de los educandos y que éstos sean capaces de auto- educarse y les brinda la posibilidad de aprender haciendo parte del proceso para que se apropien de nuevos conocimientos y a su vez interactúen con otros educandos. En la selección de las actividades de las clases sin la presencia del educador, que se orienten, se incluirán tareas que los lleven al análisis de diferentes situaciones problémicas donde tengan que hacer uso de la computadora y los asistentes matemáticos, lo que permitirá que amplíen la comprensión de los conceptos y métodos de la matemática, a partir de las cuales puedan modelar, formular definiciones y proposiciones, encontrar regularidades en la demostración de estas últimas y establecer métodos de trabajo matemático.

La evaluación responde a la pregunta ¿en qué medida se cumplen los objetivos propuestos en el modelo didáctico, con el uso de las TIC, para la formación matemática? Incluye la autoevaluación, la evaluación grupal y la del educador que se realizará mediante indicadores como los siguientes: interés mostrado en su preparación, dedicación al estudio del tema, preparación en las clases sin la presencia del educador con la guía didáctica y el sitio web, participación en las clases con la presencia del educador, nivel de desarrollo de los 
conocimientos y habilidades matemáticas mediante la solución de las tareas orientadas y la entrega de trabajos por correos a través de la red ${ }^{1}$.

Forma parte también del modelo didáctico un clima de aprendizaje que propicie el diálogo y el trabajo colaborativo. Los educadores deben propiciar que los educandos no solo desarrollen su pensamiento sino también su esfera afectiva, es decir, que lo aprendido adquiera un significado y un sentido personal tal que garantice los conocimientos para próximos aprendizajes necesarios en su desenvolvimiento en la vida.

La implementación se manifiesta en la descripción de un modelo didáctico para la formación matemática, que se auxilia del sitio web de manera que se ilustra la aplicación mediante el tema de integrales unidimensionales.
El sitio web se utiliza para mostrar información, imágenes, esquemas, manipular asistentes matemáticos, hacer consultas a través de la navegación por la bibliografía digitalizada, resolver ejercicios interactivos en una multimedia de autoevaluación, estudiar presentaciones digitales de las conferencias, clases del tema, y estudiar mediante los videos interactivos de ejercicios resueltos con y sin el empleo de los asistentes matemáticos. Contiene una página principal en la que se encuentran distribuidas las páginas de enlace a otras temáticas, por lo que, en el mismo, pueden ser consultados por educadores y educandos las páginas que lo conforman.

Esta interfaz es lo primero que aparece al acceder al sitio Integrales Unidimensionales:

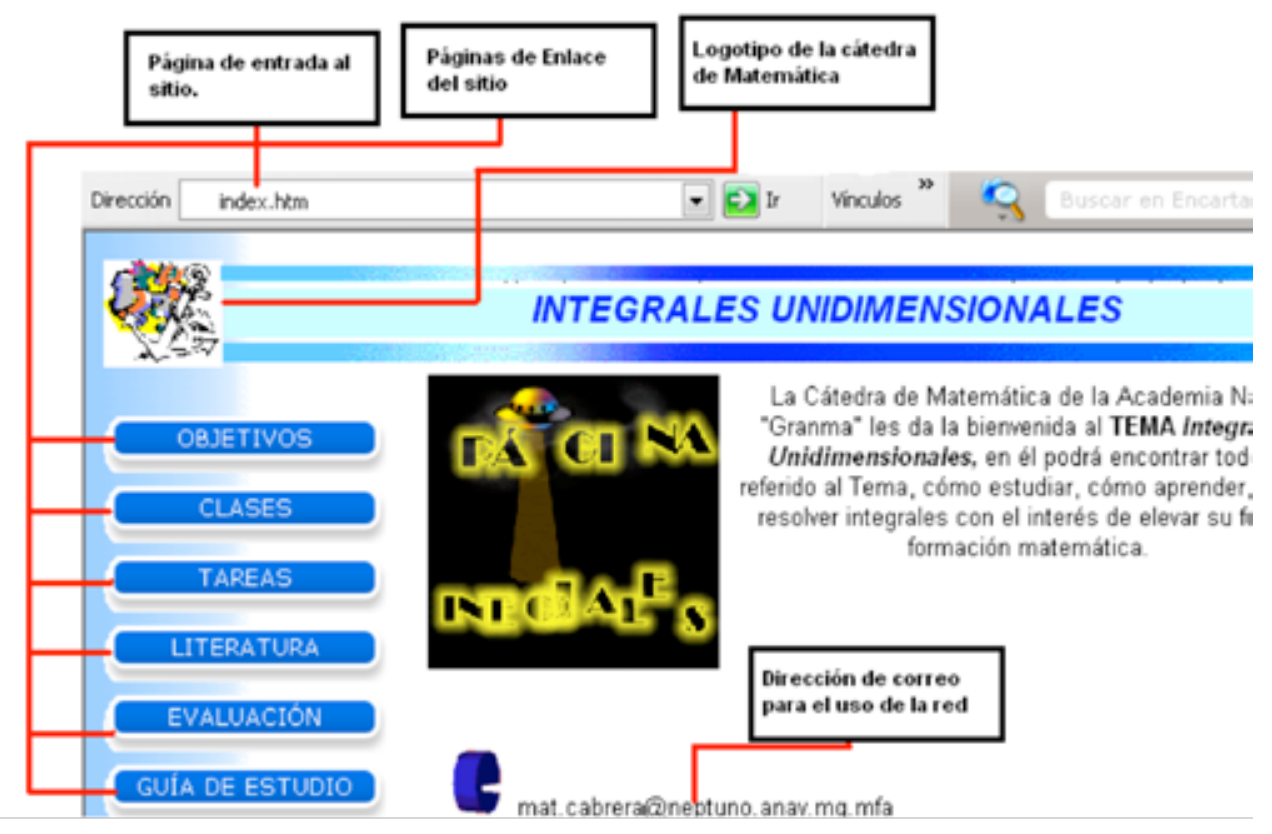

\section{¿Cómo integrar el sitio web en las clases del tema?}

En el tema integrales unidimensionales puede ser integrado el sitio web desde la Conferencia introductoria hasta el Trabajo de control parcial mediante la realización de diversas actividades que propicien la comunicación, el análisis, la reflexión, la realización de observaciones, el intercambio de criterios, el análisis de bibliografías.
La información recopilada permitió obtener una amplia gama de valoraciones con respecto a la propuesta y su correspondencia con las necesidades identificadas a partir del modelo de actuación, y la importancia del tema en la asignatura.

${ }^{1}$ Cada educando tiene asignada una colección de problemas personalizada y envía la respuesta según su dirección. 
El análisis se realiza a partir de la autovaloración para enfrentar problemas de la vida práctica en que necesiten los conocimientos, hábitos y habilidades y tengan que hacer uso de los medios de programas educativos ubicados en el sitio web. De la encuesta se pudo comprobar que:

- El 97,1\% de los encuestados valora como útil la página en su aprendizaje, el 2,9\% de regular.
- El 88,5\% de los encuestados en las dos variantes ha encontrado como ventajas de la utilización del sitio en el tema de integrales el fácil manejo, la calidad de los videos, el uso del correo, conocer el estado de sus evaluaciones, investigar en todos los archivos e informaciones que se encuentran a disposición en sus "páginas”.

En la tabla se muestran los resultados por porcentajes en las variantes de experimentación aplicadas:

TABla 1.

Resultados por porcentajes en las variantes de experimentación aplicadas

\begin{tabular}{|c|c|c|c|}
\hline \multicolumn{2}{|r|}{ ELEMENTOS A VALORAR } & $\begin{array}{l}\text { AÑO DE INST. } \\
\text { 05-06 }\end{array}$ & $\begin{array}{l}\text { AÑO DE INST. } \\
\text { 07-08 }\end{array}$ \\
\hline \multirow{4}{*}{$\begin{array}{l}\text { Medios de programas } \\
\text { utilizados para elevar } \\
\text { el autoaprendizaje }\end{array}$} & Utilizaron el correo para la entrega de trabajos & $\begin{array}{c}13 \\
(86,6 \%)\end{array}$ & $\begin{array}{c}19 \\
(95 \%)\end{array}$ \\
\hline & $\begin{array}{l}\text { Utilizaron los videos interactivos para el estudio } \\
\text { del tema }\end{array}$ & $\begin{array}{c}12 \\
(80,0 \%)\end{array}$ & $\begin{array}{c}18 \\
(90,0 \%)\end{array}$ \\
\hline & $\begin{array}{l}\text { Utilizaron los materiales didácticos para elevar } \\
\text { sus conocimientos }\end{array}$ & $\begin{array}{c}14 \\
(93,3 \%)\end{array}$ & $\begin{array}{c}20 \\
(100 \%)\end{array}$ \\
\hline & $\begin{array}{l}\text { Emplearon el asistente Derive para dar solución } \\
\text { a problemas de la especialidad }\end{array}$ & $\begin{array}{c}15 \\
(100 \%)\end{array}$ & $\begin{array}{c}20 \\
(100 \%)\end{array}$ \\
\hline
\end{tabular}

Además, al introducir el sitio web "Integrales Unidimensionales" en las clases, se observaron las siguientes consecuencias que se expresan de la manera siguiente:

La utilización del método de trabajo independiente por parte de los educandos fue adecuada.

- Se alcanzan valores elevados con el uso de videos, el empleo de tutoriales y multimedia de autoevaluación para la formación matemática.

- Se amplían y profundizan en algunos contenidos de los temas.

- Se obtienen resultados superiores al incorporar el uso del Derive y el desarrollo de habilidades en su manejo.

- Se eleva el desarrollo del pensamiento intuitivo y deductivo.

- Se desarrollan las habilidades de exponer y defender trabajos.
- Se logra un mayor desarrollo de la habilidad modelar.

\section{Conclusiones}

1. El uso de las TIC para elevar la calidad del proceso docente educativo es una necesidad didáctica actual $y$, en especial, para la enseñanza de la matemática superior.

2. El modelo didáctico se basa en la concepción de la matemática para el ingeniero como el lenguaje mediante el cual puede hacer sus representaciones; la consideración de la aplicabilidad de la matemática superior; el reconocimiento del autoaprendizaje; la presentación temático-situacional del material didáctico y la enseñanza de la matemática sobre una base histórica culturalista.

3. Un modelo didáctico, con el uso de las TIC, para la formación matemática incluye los componentes del proceso docente educativo y resalta: 
- Objetivo y contenidos de la asignatura con el empleo de las TIC.

- Ejercicios graduados según el contenido.

- El trabajo independiente como método principal.

- El predominio de las clases sin la presencia del educador.

- Variadas formas evaluativas.

- Un sitio web como soporte material del modelo.

4. El experimento pedagógico desarrollado, en sus dos variantes experimentales, corrobora que el modelo didáctico mejora la formación matemática y demuestra que los cambios significativos en los modos de actuar de los sujetos del proceso de enseñanza no pueden esperarse en un breve tiempo, ni con modificaciones parciales. El uso del modelo didáctico tendrá mayor efectividad en la medida en que se aplique para toda la asignatura y que tenga una presencia significativa en la evaluación del aprendizaje.

\section{REFERENCIAS}

Bautista, M.; Martínez, A. \& Hiracheta, R. (2014). El uso de material didáctico y las tecnologías de información y comunicación (TIC’s) para mejorar el alcance académico. Ciencia y Tecnologia, 14, 183-194.

Colectivo de Autores. (2009). Introducción de la computación en el proceso de enseñanza de la matemática superior de la Academia Naval "Granma”, Cátedra de matemática, Academia Naval, La Habana.

Delgado, J. (2009). La enseñanza de la matemática en el umbral del siglo XXI, Texto en formato digital.

Del Río, L.; González, A. \& Bucaril, N. (2014). Congreso Iberoamericano de Ciencia, Tecnología, Innovación y Educación. La integración de las TIC en las clases de matemática en el nivel universitario: ¿Cómo afrontar este desafío? Artículo 612. Buenos Aires, Argentina.

González, C. (2013). Cartilla TIC para la enseñanza de las matemáticas. Ponencia presentada en el I Congreso de Educación Matemática de América Central y El Caribe. Santo Domingo, República Dominicana.

Lovos, E.; Gibelli, T.; Saldivia, A.; Suárez, P. \& Bertone, R. (2015). Estrategia didáctica usando TICs para la enseńanza y el aprendizaje de la programación lineal en carreras de agronomía y sistemas. Corrientes, 409-417.
Maraza, B. (2016). Aprendiendo a aprender matemáticas, con ayuda de las TIC. Consultado en: https://www.educaciontrespuntocero.com/experiencias/aprendiendo-a-aprender-matematicas-con-ayuda-de-las-tic/32625.html

Marcilla, C. (2013). Las TIC en la didáctica de las matemáticas. Consultado en: http://riubu.ubu.es/bitstream/10259.1/182/1/ Marcilla_de_Frutos.pdf

Méndez, N. (2016). Modelo teórico- didáctico tecnológico para el aprendizaje de las matemáticas en la formación básica secundaria. Itinerario Educativo, 68, 65-77.

Mojica, N. (2015). El impacto de las TICs en la enseñanza de las matemáticas. Consultado en: https://prezi.com/dd-jvfbevs $1 \mathrm{~m} /$ el-impacto-de-las-tics-en-la-ensenanza-de-las-matematicas/

Murcia, E. \& Córdoba, H. (2009). Uso de las TICs y objetos de aprendizaje para la enseñanza de las Matemáticas en la UCPR. Entre Ciencia e Ingeniería, 3 (6), 129-149.

Olivier, O.; Díaz, J. \& Alonso, L. (2016). Modelo didáctico de la dinámica del proceso de enseñanza - aprendizaje de la matemática con el uso de las TIC. Didasc@lia: Didáctica y Educación, VII (3), 23-34.

Roa, N. (2013). Uso de herramientas tecnológicas en el aprendizaje de las matemáticas. Inventum, (14), 35-43.

Téliz, F. (2015). Uso didáctico de las TIC en las buenas prácticas de enseñanza de las matemáticas. Estudio de las opiniones y concepciones de docentes de educación secundaria en el departamento de Artigas. Cuadernos de Investigación Educativa, 6 (2).

Vitale, A. (2015). Una estrategia de evaluación del aprendizaje para la matemática superior en la Academia Naval "Granma", Tesis en opción al grado científico de Doctor en Ciencias Pedagógicas, Academia de las FAR, La Habana.

Rodríguez, J. (2006). Una propuesta metodológica para la utilización de las tecnologías de la Información y las Comunicaciones en el proceso de enseńanza-aprendizaje de las funciones matemáticas, Tesis en opción al grado científico de doctor en ciencias pedagógicas, Extraído de CD EduTesis, Biblioteca digital de Tesis de Maestría y doctorados en Ciencias de la Educación, Centro de documentación del ISP de Pinar del Río.

Sierra, R. (2004). Un modelo teórico para el diseño de una estrategia pedagógica en la educación primaria y secundaria básica, Tesis en opción al grado científico de doctor en ciencias pedagógicas 2004, Extraído de CD EduTesis, Biblioteca digital de Tesis de Maestría y doctorados en Ciencias de la Educación, Centro de Documentación del ISP de Pinar del Río. 
